\title{
The Importance of Communication between Healthcare Professionals: Findings from an Incident Reporting System
}

\author{
Jurgen M.J. Piek ${ }^{1}$, Iris Koppenol ${ }^{2}$,Vera Molenaar ${ }^{3}$, Olga Husson ${ }^{4}$ \\ 1. Catharina hospital. Department of Obstetrics and Gynaecology. Michelangelolaan 2, \\ 5623 EJ Eindhoven, The Netherlands \\ 2. University of Tilburg. Department of CoRPSCenter of Excellence.Warandelaan 2, \\ 5037 AB, Tilburg, The Netherlands \\ ${ }^{3 .}$ Elisabeth-TweeStedenhospital.Department of Health Care Advisors. Hilvarenbeekseweg 60, \\ 5022 GC, Tilburg, The Netherlands \\ ${ }^{4 .}$ Radboud University Medical Center, Department of Medical Psychology. Geert Grooteplein Zuid \\ 10, 6525 GA, Nijmegen, The Netherlands
}

\begin{abstract}
Objective: to assess and categorize low and medium risk incidents in an online database on 'communication' on a hospital level.

Methods: in this study we assessed and analysed all data from a Dutch Safety Management System (SMS) on the classification of 'communication'. All communication related incidents in the SMS system from January first to December 2014 on subjects: documentation, communication and organization were retrieved from the SMS database and assessed.

Results: failure in communication between healthcare professionals are most often reported (32\%). 23\% of all "communication" related incidents were misclassified as communication related and 18\% of the incidents were related to incorrect filled out forms.

Conclusions: We found that incidents on failure in communication between healthcare professionals are most often reported, reflecting the need for more standardized communication methods on the work floor. Furthermore, a high percentage of incidents were not communication related reflecting the need of instruction in the Safety Management System.
\end{abstract}

Keywords: Safety Management System, communication, incidents

\section{INTRODUCTION}

Systems for incidents reporting in hospitals ensure that information is obtained on how healthcare can be improved to enhance patient safety [1]. Since 2008 the Safety Management System (SMS) is designed and utilised for hospitals in The Netherlands [2]. In this online system, all incidents and near misses are reported by healthcare professionals and classified according to risk (low, medium, high) and are then analysed in order to achieve improvement and prevent recurrence of these incidents. However, this analysis for low and medium risk incidents does happen on the department level but not on a hospital wide level.

To gain more insight in the hospital-wide improvement potential from the lower risk classifications in SMS, we investigated the similarities between incidents classified as low and medium risk.We focussed on an important component of patient safety: communication. Problems in communication are an important cause of (severe) incidents in patient safety [3-5]. In this study we subcategorised all incidents that were reported as being communication related.

\section{Materials AND Methods}

This study conformed to the Dutch Good Clinical Practice guidelines, ethical approval was not required since no patient data was available to the researchers. All incidents reported from January 
first to December $31^{\text {st }} 2014$ on subjects: documentation, communication and organization were retrieved from the SMS database from a secondary referral hospital. In total 3.866 blinded reports, derived from 80 departments of this hospital, were analysed. We systematically studied the reports for similarities on the level of nature and root causes. These similarities were identified by two reviewers and the following 10 subcategories emerged:

- Failure in appointments between professionals (e.g. problems with handover, unavailability or absence of a doctor, not clear where the responsibility lies, no clear protocols or noncompliance to protocols)

- Communication with/to the patient (e.g. giving the wrong or no information leaflet)

- Wrong location (e.g. patient has an appointment at a certain location (the hospital has more than one physical location), but appears at another location of the hospital)

- Incorrect form/completed application (e.g. medication prescription is not clearly written)

- Appointment not/incorrectly booked (e.g. no appointment booked or with wrong doctor)

- Appointment not/incorrectly transmitted (e.g. patient has not been informed on the appointment or are present at the wrong time)

- Wrong patient data

- Problems with insurance/ patient ID

- Missing documents

- No communication related report (nature of incident was mistakenly grouped under 'communication' incidents)

All departments have been analysed and the reports were posted in one of the ten categories by one of the reviewers (IK). Subsequently, the reports were taken by the other evaluator (JP), in order to come to the final categorization. There were also reports (84) that could not be categorized by insufficient or unclear information.

\section{ReSUlts}

Disregarding the 'no communication related report' category (954 reports, $23 \%$ of the total number of reports), the number of communication reports was 2.912. Table 1 lists the categories listed with the corresponding number of reports. Failure in appointments between professionals was found in $31.65 \%$ of all communication related incidents, followed by incorrect form/incorrect completed application in $18.11 \%$ of all incidents. Table 2 shows the departments that reported most incidents. Most incidents $(12.96 \%)$ were posted by appointment office officers.

Table 1. Number and percentage of incident reports per subcategory

\begin{tabular}{|l|l|}
\hline Category & Number of incident reports (\%) \\
\hline Failure in appointments between professionals & $1.197(31.65 \%)$ \\
\hline No communication related report & $870(23.00 \%)$ \\
\hline Incorrect form/completed application & $685(18.11 \%)$ \\
\hline Appointment not/incorrectly booked & $362(9.57 \%)$ \\
\hline Wrong location & $357(9.44 \%)$ \\
\hline Appointment not/incorretly transmitted & $142(3.75 \%)$ \\
\hline Communication with/to the patient & $78(2.06 \%)$ \\
\hline Wrong patient data & $67(1.77 \%)$ \\
\hline Problems with insurance/ patient ID & $18(0.48 \%)$ \\
\hline Missing documents & $6(0.16 \%)$ \\
\hline
\end{tabular}

Incident reports on the subject of documentation, communication and organization were systematically studied for similarities on the level of nature and root causes. By identifying these similarities, 10 subcategories emerged and were detected in the stated percentage of the reports. 
The Importance of Communication between Healthcare Professionals: Findings from an Incident Reporting System

Table 2. Top nine departments with most incident reports and percentages*

\begin{tabular}{|l|l|}
\hline Department & Number of incident reports (\%) \\
\hline Appointment office & $490(12.96 \%)$ \\
\hline Surgical department & $141(3.73 \%)$ \\
\hline Orthopedic/traumatology department & $131(3.46 \%)$ \\
\hline Intensive Care Unit & $130(3.44 \%)$ \\
\hline Operating floor & $129(3.41 \%)$ \\
\hline Geriatrical department & $129(3.41 \%)$ \\
\hline Radiology & $127(3.36 \%)$ \\
\hline Department of cardiology & $115(3.04 \%)$ \\
\hline Department of pulmonology & $105(2.78 \%)$ \\
\hline
\end{tabular}

*Excluding the category 'no communication related report'

Number (and percentage) of incident reports on the subject documentation, communication and organization of the nine departments reporting the most.

\section{DisCUSSION}

To our knowledge, this is the first time all low and medium risk incident reports on the subject of communication on a hospital level have been analysed systematically. In this secondary referral hospital 'failure in appointments between professionals' was the most common communication related incident in the lower risk classification group. Mostly, these incidents have minor impact on quality of healthcare. However, it is likely that patients lose trust in the healthcare system if these incidents happen regularly [6]. This also creates support for the observation that more clear communication between professionals can potentially result in less incidents. A great part of this can be achieved by standardizing handovers with guidelines for the exchange of information, supplementing oral transmission with written transfer and well-developed information systems between healthcare providers [7, 8]. One such tool is the SBAR method [5].

The category 'incorrect form/completed application' contains the second most reported incidents. A meta-study concerning correct documentation of caregiving of nurses led to seven themes to improve the quality of nursing documentation. This includes patient centred communication, documentation in a logical and sequential manner, documentation at the time events occur, and accurate, objective and comprehensive documentation of the condition of and care given to a patient following legal requirements for documentation [9]. However, this should count for all care givers involved. In a study at adepartment of radiology, errors in requests and reports led to communication problems and a reduced quality of patient care [10]. The implementation of information technology (IT) can improve the quality of care by improving administrative and identification information [10].

Our analysis shows that appointment related incidents are rather common $(22.76 \%)$ and that most incidents were reported at the appointment office. This might be caused by the digital system in which appointments are made and the high risk of human failure in this. This incident subcategory might be improved by a text message system by which patients receive a message before their appointment [11, 12]. This reminder tool seems to be effective to reduce the number of patients failing to attend their appointments and thus will possibly reduce the number of incident reports. To reduce the risk of human failure in making the appointment and communicating it to the patient, direct printouts of the appointment or an e-mail to the patient, in contrast to a written appointment card, could be a possible solution.

A large percentage $(23 \%)$ of all incidents were not communication related. This indicates a need for instruction in the SMS system. Alternatively, this might be due to the fact that in the SMS system one can choose from quite a few categories. As reporter it sometimes is difficult to pinpoint the exact category. A solution would be to decrease the number of categories.

We realise that the drawback of our study is that the quality of input of the data has an important effect on the quality of the output. In this kind of studies one is dependent on the willingness to report incidents. The fact that over the last five years the reported incidences in the communication category were rather steady reassures in this matter. 
In conclusion, incidents on communication between professionals are most commonly reported in our study reflecting the need for a more standardized communication method within hospitals. A large percentage of incidents are wrongly classified indicating the need for training in the use of the SMS system.

Disclosure of interest: no disclosures.

Contribution to Authorship IK and JP: data assessment. IK, JP, OH and VM: drafting the article.

No funding was available for this study.

Ethical approval for this study was not required.

\section{REFERENCES}

[1] Stavropoulou C, Doherty C, Tosey P. How Effective Are Incident-Reporting Systems for Improving Patient Safety? A SystematicLiterature Review. MilbankQuarterly, 2015, 93(4), 826866.

[2] Noord IV, Zwijnenberg N, Wagner C. Patiëntveiligheidscultuur in Nederlandse Ziekenhuizen: een stap in de goede richting?. 2013.

[3] Heideveld-Chevalking AJ, Calsbeek H, Damen J, Gooszen H, Wolff AP. The impact of a standardized incident reporting system in the perioperative setting: a single center experience on 2,563 'near-misses' and adverse events. Patient safety in surgery, 2014, 8(1), 1.

[4] Merten H, Lubberding S, Wagtendonk I, Johannesma PC, Wagner C. Patient safety in elderly hip fracture patients: design of a randomised controlled trial. BMC health services research, 2011, 11(1), 1.

[5] Randmaa M, Mårtensson G, Swenne CL, Engström M. SBAR improves communication and safety climate and decreases incident reports due to communication errors in an anaesthetic clinic: a prospective intervention study. BMJ open, 2014, 4(1), e004268.

[6] Kuzel AJ, Woolf SH, Gilchrist VJ, Engel JD, LaVeist TA, Vincent C, Frankel RM. Patient reports of preventable problems and harms in primary health care. The Annals of Family Medicine, 2004, 2(4), 333-340.

[7] Arora VM, Manjarrez E, Dressler DD, Basaviah P, Halasyamani L, Kripalani S. Hospitalist handoffs: a systematic review and task force recommendations. Journal of hospital medicine, 2009, 4(7), 433-440.

[8] Meijboom BR, Bakx SJ, Westert GP. Continuity in health care: lessons from supply chain management. The International journal of health planning and management, 2010, 25(4), 304317.

[9] Jefferies D, Johnson M, Griffiths R. A meta-study of the essentials of quality nursing documentation. International journal of nursing practice, 2010, 16(2), 112-124.

[10] Pirnejad H, Niazkhani Z, Bal R. Clinical communication in diagnostic imaging studies: mixedmethod study of pre-and post-implementation of a hospital information system. Applied clinical informatics, 2013, 4(4), 541.

[11] Downer SR, Meara JG, Da Costa AC, Sethuraman K. SMS text messaging improves outpatient attendance. Australian Health Review, 2006, 30(3), 389-396.

[12] Da Costa TM, Salomão PL, Martha AS, Pisa IT, Sigulem D. The impact of short message service text messages sent as appointment reminders to patients' cell phones at outpatient clinics in Sao Paulo, Brazil. International journal of medical informatics, 2010, 79(1), 65-70. 